\title{
Role of Rhodopsin and Arrestin Phosphorylation in Retinal Degeneration of Drosophila
}

\author{
Inga Kristaponyte, Yuan Hong, Haiqin Lu, and Bih-Hwa Shieh \\ Department of Pharmacology, Center for Molecular Neuroscience and Vision Research Center, Vanderbilt University, Nashville, Tennessee 37232
}

\begin{abstract}
Arrestins belong to a family of multifunctional adaptor proteins that regulate internalization of diverse receptors including G-proteincoupled receptors (GPCRs). Defects associated with endocytosis of GPCRs have been linked to human diseases. We used enhanced green fluorescent protein-tagged arrestin 2 (Arr2) to monitor the turnover of the major rhodopsin (Rh1) in live Drosophila. We demonstrate that during degeneration of norpA ${ }^{P 24}$ photoreceptors the loss of Rh1 is parallel to the disappearance of rhabdomeres, the specialized visual organelle that houses Rh1. The cause of degeneration in $\operatorname{norpA}^{P 24}$ is the failure to activate CaMKII $\left(\mathrm{Ca}^{2+} / \mathrm{calmodulin}\right.$-dependent protein kinase II) and retinal degeneration C (RDGC) because of a loss of light-dependent $\mathrm{Ca}^{2+}$ entry. A lack of activation in CaMKII, which phosphorylates Arr2, leads to hypophosphorylated Arr2, while a lack of activation of RDGC, which dephosphorylates Rh1, results in hyperphosphorylated Rh1. We investigated how reversible phosphorylation of Rh1 and Arr2 contributes to photoreceptor degeneration. To uncover the consequence underlying a lack of CaMKII activation, we characterized ala $^{l}$ flies in which CaMKII was suppressed by an inhibitory peptide, and showed that morphology of rhabdomeres was not affected. In contrast, we found that expression of phosphorylation-deficient Rh1s, which either lack the C terminus or contain Ala substitution in the phosphorylation sites, was able to prevent degeneration of norpA ${ }^{P 24}$ photoreceptors. This suppression is not due to a loss of Arr2 interaction. Importantly, co-expression of these modified Rh1s offered protective effects, which greatly delayed photoreceptor degeneration. Together, we conclude that phosphorylation of Rh1 is the major determinant that orchestrates its internalization leading to retinal degeneration.
\end{abstract}

\section{Introduction}

In Drosophila, mutations affecting morphogenesis or signaling of photoreceptors have detrimental consequences leading to the demise of photoreceptors (for review, see Shieh, 2011). Particularly, mutations that perturb the biosynthesis, transport, and deposition of the major rhodopsin, rhodopsin 1 (Rh1) constitute one of the major causes of retinal degeneration. Rh1 is the major rhodopsin found in R1-6 photoreceptors of the compound eye (O'Tousa et al., 1985; Zuker et al., 1985). Activated rhodopsin (metarhodopsin) is coupled to the heterotrimeric Gq protein, leading to increased activity of phospholipase $\mathrm{C} \beta$ (PLC $\beta$ ) encoded by the no-receptorpotential (norpA) gene. PLC $\beta$ catalyzes the breakdown of phosphatidylinositol 4,5-bisphosphate to release diacylglycerol, which generates the second messenger, possibly polyunsaturated fatty acids (Leung et al., 2008), to gate the transient receptor potential (TRP) and TRP-like channels (Hardie, 2007). Subsequently, the opening of these two channels brings about an influx of $\mathrm{Ca}^{2+}$ and $\mathrm{Na}^{+}$that

Received Feb. 6, 2012; revised June 9, 2012; accepted June 14, 2012.

Author contributions: B.-H.S. designed research; I.K., Y.H., H.L., and B.-H.S. performed research; I.K., H.L., and B.-H.S. analyzed data; I.K. and B.-H.S. wrote the paper.

This work is supported by NIH Grants R01 (B.-H.S.) and P30 EY08126 to Vanderbilt University. We thank Drs. Charles Zuker and Steve Britt for the fly stocks and Dr. Rama Ranganathan for the YC4 vector. We thank Dr. Chang Chung for invaluable assistance with fluorescence microscopy.

Correspondence should be addressed to Bih-Hwa Shieh, Department of Pharmacology, Center for Molecular Neuroscience and Vision Research Center, 402 RRB, Vanderbilt University, Nashville, TN 37232. E-mail: bih-hwa.shieh@vanderbilt.edu.

DOI:10.1523/JNEUROSCI.0565-12.2012

Copyright $\odot 2012$ the authors $\quad 0270-6474 / 12 / 3210758-09 \$ 15.00 / 0$ depolarizes photoreceptors (for review, see Pak and Leung, 2003; Wang and Montell, 2007; Shieh, 2011).

A rise of cytoplasmic $\mathrm{Ca}^{2+}$ serves to modulate $\mathrm{Rh} 1$ by turning on both CaMKII $\left(\mathrm{Ca}^{2+} /\right.$ calmodulin-dependent protein kinase II) and RDGC (retinal degeneration C). RDGC is a $\mathrm{Ca}^{2+}$ dependent protein phosphatase that catalyzes dephosphorylation of phosphorylated Rh1 (Byk et al., 1993; Vinós et al., 1997; Kiselev et al., 2000), while activated CaMKII phosphorylates arrestin 2 (Arr2; Dolph et al., 1993; Matsumoto et al., 1994; Kahn and Matsumoto, 1997), which is the major arrestin critical for the inactivation of Rh1 (Dolph et al., 1993). In norpA $A^{P 24}$, these two $\mathrm{Ca}^{2+}$-dependent mechanisms fail to be switched on, and activated Rh1 cannot be inactivated. Instead, activated Rh1 forms a stable complex with Arr2 (Alloway et al., 2000; Orem and Dolph, 2002; Chinchore et al., 2009), resulting in retinal degeneration. It remains to be elucidated how the absence of CaMKII and/or RDGC contributes to the formation of the stable Rh1/Arr2 complex and its internalization. One of the prevailing hypotheses suggests that a loss of phosphorylation in Arr2 promotes endocytosis, as unphosphorylated Arr2 interacts strongly with clathrin in vitro (Kiselev et al., 2000).

We used microscopy to monitor the degeneration of norpA $A^{P 24}$ photoreceptors via enhanced green fluorescent protein (eGFP)tagged (Yang et al., 1996) Arr2. We demonstrate that the progressive deterioration of rhabdomeres is parallel to the decrease of $\mathrm{Rh} 1$, supporting the understanding that Rh1 plays a critical role in the survival and maintenance of photoreceptors (Leonard et al., 1992; Kumar and Ready, 1995). We show that modified Arr2 mimicking CaMKII phosphorylation (Arr2 ${ }^{\mathrm{S} 366 \mathrm{D}}$ ) interacts with 
Rh1 in vitro and in vivo and displays similar subcellular distribution. Importantly, suppression of Arr2 phosphorylation upon inhibition of CaMKII fails to negatively impact the morphology of rhabdomeres. It is of interest to note that, unlike vertebrate visual arrestins, Arr2 interaction with Rh1 is independent of Rh1's phosphorylation. However, the interaction between phosphorylation-deficient Rh1 and Arr2 prevents degeneration of norp $A^{P 24}$ photoreceptors. Our results strongly support the notion that phosphorylation of Rh1, but not the lack of Arr2 phosphorylation, is required for the formation of the stable Rh1/Arr2 complex leading to degeneration of photoreceptors.

\section{Materials and Methods}

Recombinant DNA and molecular biology. Full-length arr2 cDNA was obtained by PCR using a retinal cDNA library as the template and AmpliTaq or EasyA (Life Technologies) as the enzyme. Similarly, modified cDNA corresponding to the truncated Arr2, Arr2 ${ }^{1-356}$, and Arr2 containing an Asp substitution at Ser ${ }^{366}$ was generated by site-directed mutagenesis using PCR. All cDNAs with engineered EcoRI (at $5^{\prime}$ ) and XhoI (at $3^{\prime}$ ) restriction enzyme sites were subcloned by the TA cloning into PCR2.1 (Life Technologies) and nucleotide sequences are confirmed by automatic DNA sequencing. Wild-type (wt) arr2 or arr $2^{\mathrm{S} 366 \mathrm{D}}$ was subcloned into pEGFP (Clontech) for creating a chimera gene with eGFP inserted at the $\mathrm{C}$ terminus of Arr2. The nucleotide sequences for Arr2eGFP were subsequently subcloned into the YC4 transgenic vector (kindly provided by Rama Rangananthan, University of Texas Southwestern) that contains Drosophila Rh1 promoter (Mishra et al., 2007).

Generation of ${ }^{35} S$-Arr2 by coupled transcription and translation system. Incorporation of ${ }^{35} \mathrm{~S}$-methionine into wt or modified Arr2 was accomplished by in vitro transcription and translation using the transcription and translation system (Promega) according to the manufacturer's protocol. The following were added for a standard reaction $(50 \mu \mathrm{l})$ : a plasmid $(0.2-1 \mu \mathrm{g})$ containing the full-length Arr2 cDNA in pBluescript SK vector (Agilent Technologies), ${ }^{35} \mathrm{~S}$-methionine ( $40 \mu \mathrm{Ci}$ ) (PerkinElmer), and rabbit reticulocyte lysates $(25 \mu \mathrm{l})$. Synthesis of the radiolabeled proteins was quantified by Phosphor-Imaging analysis following SDS/ PAGE. ${ }^{35}$ S-labeled Arr2 was used directly for membrane binding assays.

Metarhodopsin binding assays. Heads of 10 female flies were dissected and homogenized in $30 \mu \mathrm{l}$ binding buffer ( $150 \mathrm{~mm} \mathrm{KCl}, 20 \mathrm{~mm}$ Tris, $5 \mathrm{~mm}$ DTT, $1 \mu \mathrm{g} / \mathrm{ml}$ leupeptin, $1 \mu \mathrm{g} / \mathrm{ml}$ aprotinin, $1 \mu \mathrm{g} / \mathrm{ml}$ pepstain, 1 mm benzamide, 1 $\mathrm{mm}$ benzamidine, and $1 \mathrm{~mm}$ phenylmethylsulfonyl fluoride). Membranes were collected upon microcentrifugation, washed twice with binding buffer $(100 \mu \mathrm{l})$, and subject to treatment with either blue or orange light for $4 \mathrm{~min}$. Light-treated membranes were incubated for $20 \mathrm{~min}$ with ${ }^{35} \mathrm{~S}$-Arr2 upon addition of $10 \mu \mathrm{l}$ probe and $15 \mu \mathrm{l}$ binding buffer at room temperature. Membranes from arr21 were used to reduce the competition of Rh1 binding by the endogenous Arr2 while membranes from ninaE $E^{I 17}$ that lack endogenous Rh1 were used as a negative control. Following metarhodopsin binding, membrane-bound ${ }^{35}$ S-Arr2 was collected following centrifugation and washed with binding buffer three times. The recovered ${ }^{35} \mathrm{~S}$-Arr2 was analyzed by SDS/PAGE followed by autoradiography or Phosphor-Imaging analysis (GE Healthcare).

Generation of anti-Arr2 antibodies. Polyclonal antibodies were obtained by immunizing rabbits with bacterially expressed full-length Arr2 (Biosynthesis).

Quantitative Western blotting. Fluorophore-conjugated secondary antibodies were used and the signals were visualized and quantified by the Odyssey Infrared Imaging system (LI-COR). Polyclonal antibodies against INAD (Shieh and Niemeyer, 1995) and monoclonal antibodies for Rh1 (4C5; Developmental Studies Hybridoma Bank) were used. Protein levels were expressed as a percentage of the 2-d-old flies of the same genotype.

Isolation of ommatidia from pupae. Pupae heads (stage P13-P15) of either sex were removed and transferred into a drop of $1 \times$ PBS on a microscope slide. Retinas were gently dissociated using insect needles and larger debris was removed. The preparation was immediately imaged using the Olympus AX70 microscope.

Fluorescence microscopy. $\mathrm{CO}_{2}$ anesthetized flies of either sex were immobilized in clay that was placed in a $50 \mathrm{~mm}$ Petri dish. The preparation was covered with water and examined using an upright Olympus AX70 microscope equipped with a $40 \times$ water-immersion lens (LUMPLFL $40 \times$ ). The images were acquired using IPLab image acquisition software (BioVision Technologies) and Retiga camera from QImaging. Multiple images $(n \geq 3)$ were taken for each experimental condition.

Quantitation of fluorescent deep pseudopupil and rhabdomere size. Fluorescent deep pseudopupil (fdpp) was imaged using Olympus AX70 microscope using $10 \times$ objective and the intensity was measured using ImageJ from National Institutes of Health (Bethesda, MD). The mean intensity values were calculated for each deep pseudopupil (dpp). Cross-sectional areas of rhabdomeres of R1-6 photoreceptors in six-eight representative ommatidia were quantified by ImageJ, and calculated for the mean area values.

$P$-element-mediated germ-line transformation. Recombinant YC4 constructs for expressing eGFP fusion protein containing either wt or modified arr2 cDNA were injected into $y^{1} w^{67 c 23}$ embryos (Rainbow Transgenic Flies). Multiple lines with the transgene integrated into the second or third chromosome were selected for analysis.

Drosophila stocks. Most of flies used are in white-eyed background except ala $^{l}$, which is in red-eyed background. Fly stocks were maintained at $25^{\circ} \mathrm{C}$ in a $12 \mathrm{~h}$ light/dark cycle. $\operatorname{arr} 2^{1}$ was obtained from Dr. Charles Zuker (Columbia University, NY) and transgenic flies expressing modified Rh1 (Rh1 $\Delta 356$ and Rh1CT S >A) were kindly provided by Dr. Steve Britt (University of Colorado, CO). norp $A^{P 24}$ was obtained from Dr. William Pak (Purdue University, IN) and ala from Dr. Leslie Griffith (Brandeis University, MA). Standard crosses were used to introduce nor$p A^{P 24}$ or ninaE $E^{I 17}$ into the background of transgenic lines.

Statistical analysis. Bar graph data were analyzed with GradPad Prism 4.0 software. The one-way ANOVA statistical test was applied. Data represent mean \pm SEM from at least three independent experiments $(n \geq 3)$ unless otherwise noted in the figure legends. Comparisons between control and experimental conditions were performed using a two-tailed Student's $t$ test. The differences were considered significant when $p<0.05$.

\section{Results}

The in vitro interaction between activated Rh1 and Arr 2 is not affected by Arr 2 phosphorylation

In Drosophila photoreceptors, Arr2 is the major visual arrestin critical for the inactivation of photoactivated Rh1 by a stoichiometric interaction (Dolph et al., 1993; Satoh et al., 2010). Arr2 is phosphorylated by CaMKII at Ser $^{366}$ (Matsumoto et al., 1994; Kahn and Matsumoto, 1997; Kahn et al., 1998), which has been implicated in regulating membrane dissociation of Arr2 following inactivation of photoactivated Rh1 (Alloway and Dolph, 1999). Our laboratory previously proposed that the Rh1 interaction with Arr2 is positively regulated by Arr2 phosphorylation, as reduced Arr2 phosphorylation diminishes its ability to uncouple Rh1 (Lu et al., 2009).

We explored how Arr2 phosphorylation affects the Rh1 interaction by examining two modified Arr2s. By in vitro metarhodopsin binding assays, we compared the association of ${ }^{35}$ S-labeled Arr2 with photoactivated Rh1 in the membranes prepared from fly heads. Consistent with previous reports (Alloway and Dolph, 1999; Alloway et al., 2000; Kiselev et al., 2000), we show that wt ${ }^{35} \mathrm{~S}$-Arr2 interacts specifically with activated $\mathrm{Rh} 1$ in membranes treated with blue light $(\lambda 480 \mathrm{~nm}, \mathrm{~B})$, but not with inactivated Rh1 in membranes treated with orange light $(\lambda 580$ $\mathrm{nm}, \mathrm{O}$ ) (Fig. 1, top). In contrast, we observed a reduced ${ }^{35} \mathrm{~S}$-Arr2 binding to membranes isolated from ninaE that lacks Rh1 (O'Tousa et al., 1985; Zuker et al., 1985) (Fig. 1, lane 4). Significantly, ${ }^{35} \mathrm{~S}$-Arr2 ${ }^{\mathrm{S} 366 \mathrm{D}}$ that mimicked phosphorylated Arr2 at $\mathrm{Ser}^{366}$ displayed binding specificity similar to wt ${ }^{35} \mathrm{~S}$-Arr2 (Fig. 1, middle). We also examined the contribution of the $\mathrm{C}$ terminus of Arr2 by testing Arr $2^{1-356}$ that lacks the last 45 aa including Ser ${ }^{366}$. We found that truncated Arr2 exhibited comparable binding specificity toward photoactivated Rh1 in fly head membranes (Fig. 1, bottom). 
The Rh1 interaction with Arr2 can be abolished when Rh1 becomes inactivated upon orange light treatment, which results in the dissociation of Arr2 from the membrane (Fig. 1, B $\rightarrow \mathrm{O}$, lane 2). We investigated whether phosphorylation of Arr2 negatively modulates the Rhl interaction by promoting its membrane dissociation. However, we show that both ${ }^{35} \mathrm{~S}-\mathrm{Arr} 2{ }^{\mathrm{S} 366 \mathrm{D}}$ and ${ }^{35} \mathrm{~S}$ Arr ${ }^{1-356}$ can be released from inactivated Rh1, similar to wt ${ }^{35}$ S-Arr2 (Fig. 1, lane 2). Based on these results, we conclude that phosphorylation of Arr2 does not greatly modify its association with photoactivated Rh1, nor its release from inactivated Rh1. Moreover, the $\mathrm{C}$ terminus of Arr2 is not critical for regulating either the association with or dissociation from Rh1.

\section{Both wt Arr2-eGFP and Arr2 ${ }^{\text {S366D }}$-eGFP interact with} activated Rh1 and display rhabdomeric localization in vivo To investigate the interaction between Arr2 and Rh1 in vivo, we generated transgenic flies expressing modified Arr2s containing an eGFP tag at the $\mathrm{C}$ terminus. Each of the modified Arr2 (wt and Arr $2^{\mathrm{S} 366 \mathrm{D}}$ ) is transcriptionally controlled by the Rh1 promoter and therefore is expressed only in R1-6 photoreceptors. Despite the use of a strong promoter, we show that the steady-state level of wt Arr2-eGFP or Arr2 ${ }^{\text {S366D }}$-EGFP in transgenic flies was $\sim 12.1 \pm 3.3 \%(n=3)$ of endogenous Arr2 (Fig. $2 F)$. A recent study by Ready's group showed that GFP-tagged wt Arr2 was functionally active similar to wt Arr2 (Satoh et al., 2010).

Previous studies have established that Arr2 undergoes light-dependent translocation. Arr2 is present predominantly in the cytosol, but rapidly translocates to rhabdomeres by binding to activated Rh1 upon light stimulation (Kiselev et al., 2000; Satoh and Ready, 2005; Satoh et al., 2010). Consistently, we observed in lightstimulated live retinas that both wt Arr2eGFP (Fig. 2A) and Arr2 ${ }^{\text {S366D }}$-eGFP (Fig. $2 B$ ) were highly enriched in rhabdomeres of R1-6 photoreceptors. This is likely due to the recruitment of Arr2-eGFP by activated Rh1 as blue light $(\lambda 480 \mathrm{~nm})$ that activates Rh1 also corresponds to the excitation wavelength for eGFP. To confirm the light-dependent translocation, we introduced the transgene into the ninaE $E^{I 17}$ background. We found that in the absence of Rh1, the rhabdomeric enrichment of both wt Arr2-eGFP (Fig. 2C) and Arr2 ${ }^{\text {S366D }}$-eGFP (data not shown) was eliminated.

We also examined the distribution of wt Arr2-eGFP in ommatidia (or unit eyes) isolated from either sex of flies. We show that wt Arr2-eGFP was preferentially associated with rhabdomeres of photoreceptors, which span longitudinally along the entire length of R1-6 photoreceptors (Fig 2D). In contrast, wt Arr2-eGFP exhibited a uniform cytoplasmic distribution in photoreceptors missing Rh1 (Fig. 2E). Similarly, Arr2 ${ }^{\text {S366D }}$-eGFP also exhibited the Rh1-dependent subcellular localization (data not shown). Thus, we conclude that both wt Arr2-eGFP and Arr2 ${ }^{\text {S366D }}$-eGFP translocate to rhabdomeres upon binding to activated Rh1, and phosphorylation of Arr2 at Ser ${ }^{366}$ does not modify its subcellular localization.

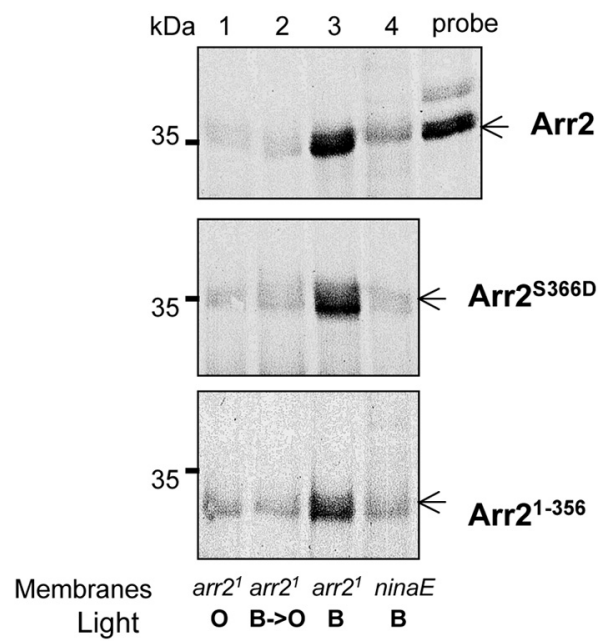

Figure 1. Association and dissociation between Rh1 and modified Arr2 in vitro. ${ }^{35} \mathrm{~S}$-Arr2 (wt) binding and release from membranes containing either activated (blue-light-treated, B) or inactivated Rh1 (orange-light-treated, 0 ) were analyzed by SDS/PAGE followed by autoradiography (top). Modified Arr2s containing Asp substitution at residue 366 mimicking phosphorylated Ser (Arr2 ${ }^{5366 \mathrm{D}}$, middle) or missing the C terminus (Arr ${ }^{1-356}$, bottom) display similar binding specificity toward activated Rh1 (lane 3), like wt Arr2. Moreover, dissociation of modified Arr2 from inactivated Rh1 (lane 2) is similar to that of wt Arr2. Binding assays were performed using membranes from arr ${ }^{1}$ that contain $10 \%$ of endogenous Arr 2 and membranes from ninaE ${ }^{177}$ were used for the negative control. A representative autoradiograph is shown.
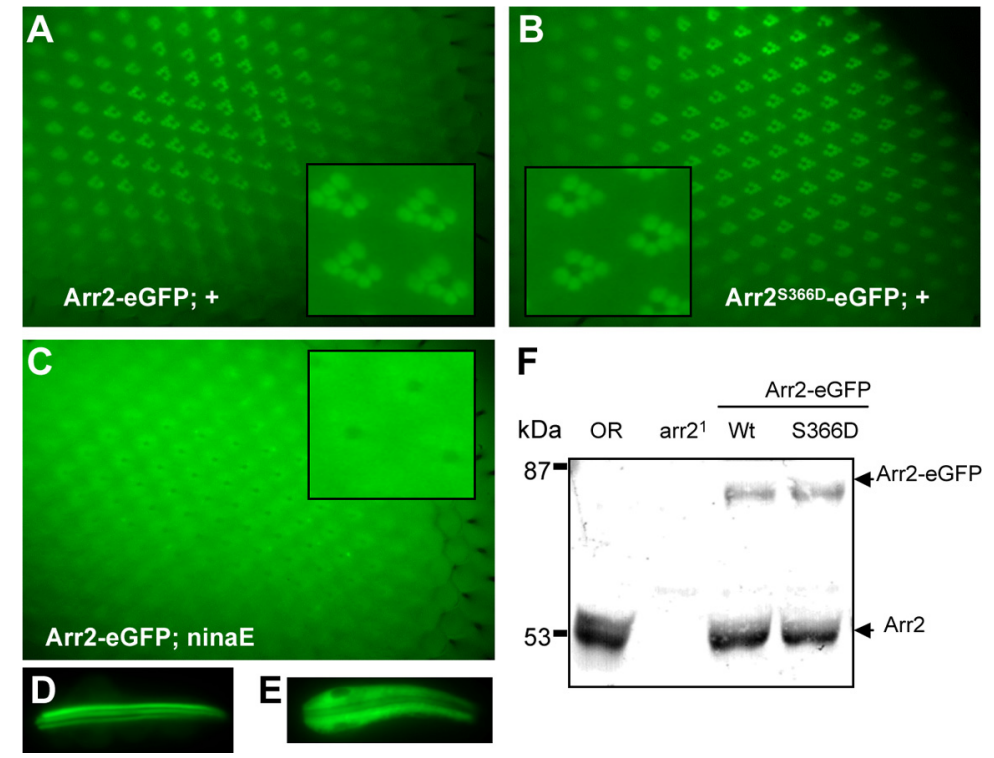

Figure 2. Interaction between activated Rh1 and wt Arr2 or Arr2 ${ }^{\mathrm{S366D}}$ in vivo. The interaction between activated Rh1 and Arr2 was investigated by fluorescence microscopy in live flies. $\boldsymbol{A}$, The distribution of wt Arr2-eGFP is predominantly in the rhabdomere of R1- 6 photoreceptors. $\boldsymbol{B}$, The subcellular localization of Arr2 ${ }^{\text {S366D }}$-eGFP is similar to that of wt Arr2-eGFP. $\boldsymbol{C}$, The rhabdomeric distribution of wt Arr2-eGFP is greatly diminished in the nina ${ }^{177}$ background lacking endogenous Rh1. D, In isolated ommatidia, wt Arr2-eGFP fusion proteins are highly concentrated in rhabdomeres, but display uniform distribution in the absence of Rh1 (ninaE) (E). $\boldsymbol{F}$, The steady-state level of both Arr2-eGFP fusion proteins is $\sim 12.1 \pm 3.3 \%(n=3)$ of endogenous Arr2 by Western blotting. Three-day-old flies of various genotypes were used for the microscopic and Western analyses.

Loss of Rh1 is parallel to the reduction of rhabdomeres during the progression of retinal degeneration in live norp $A^{P 24}$ flies

We investigated the proximal events leading to degeneration of norp $A^{P 24}$ photoreceptors. It was proposed that in the degenerating norp $A^{P 24}$ photoreceptors Arr2 forms a stable complex with photoactivated Rh1, and the massive endocytosis of the Rh1/Arr2 

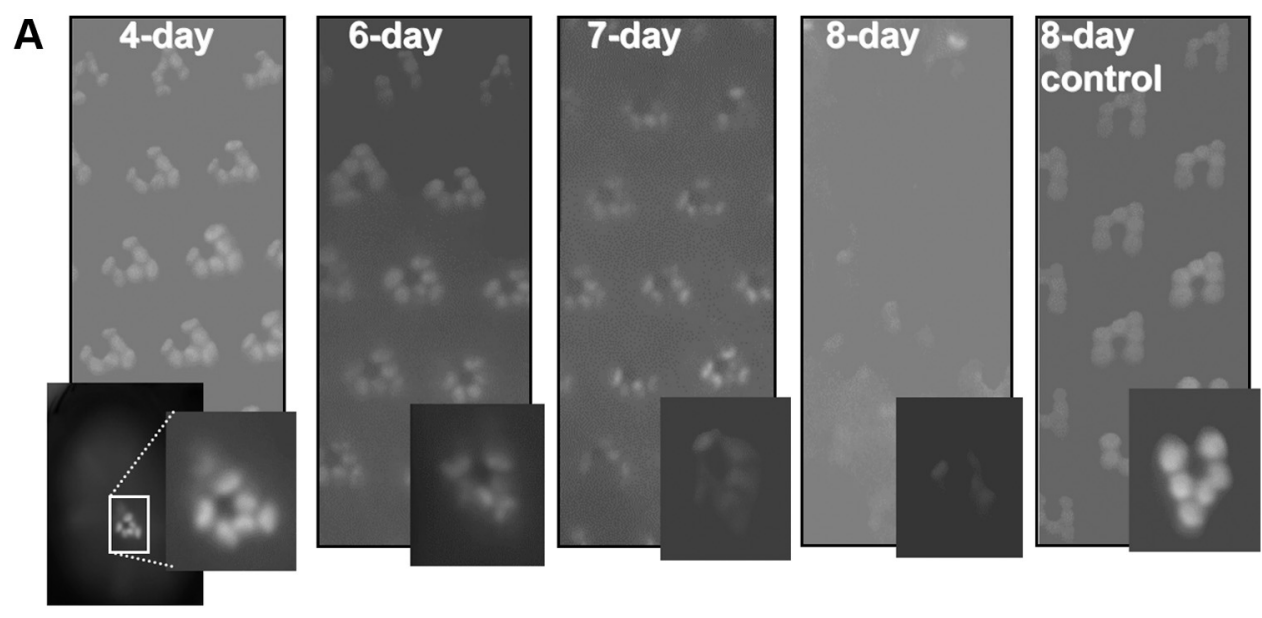

B

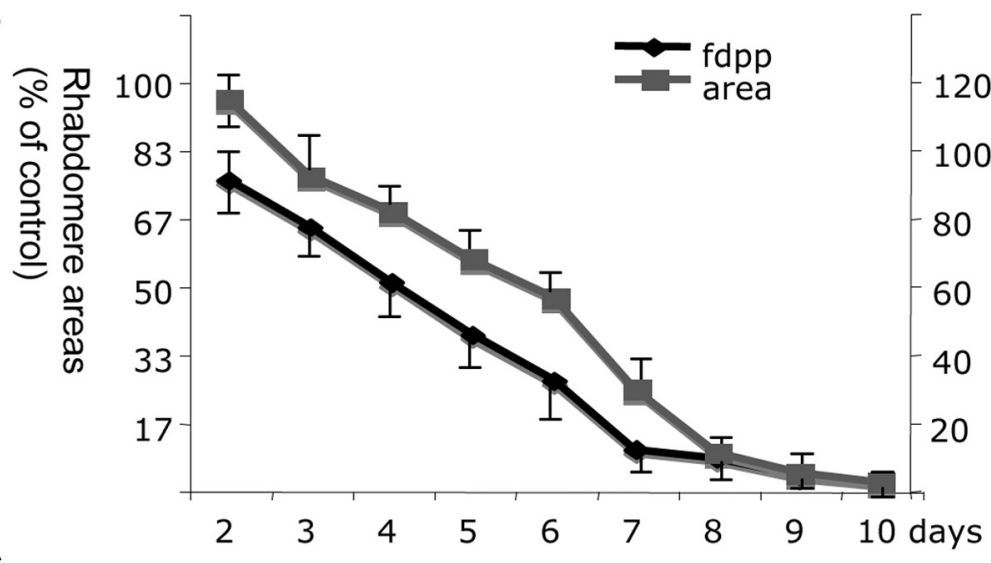

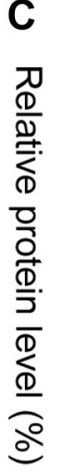

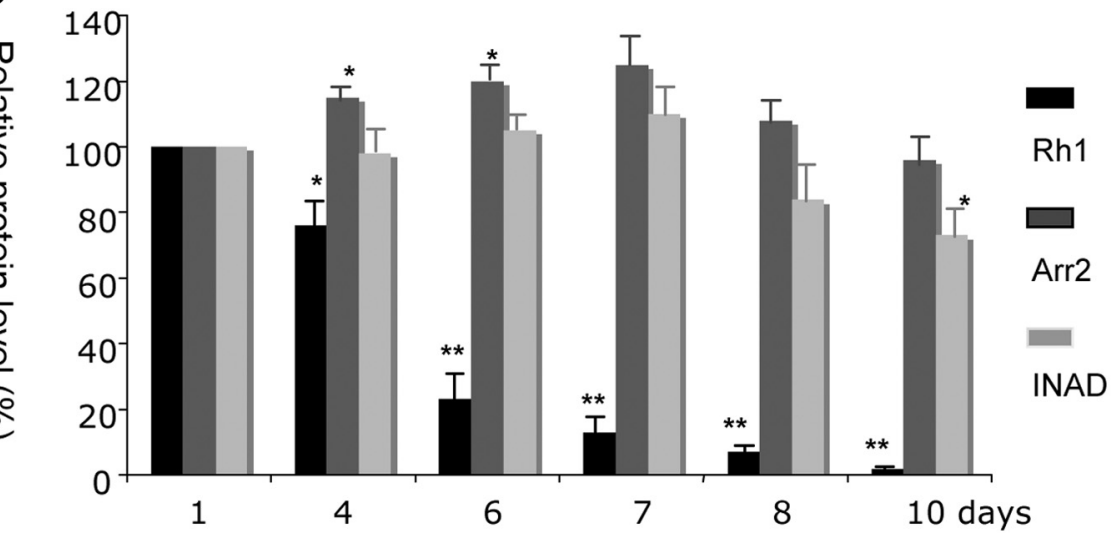

Figure 3. Progression of retinal degeneration in live retinas of norp $A^{P 24}$ photoreceptors. $A$, Degeneration was characterized by the age-dependent deterioration of rhabdomeres in $\mathrm{R} 1-6$ photoreceptors. Rhabdomeres are visualized by wt Arr2-eGFP that binds to activated Rh1. An almost complete loss of wt Arr2-eGFP is evident at 8-9 d posteclosion. Insets depict fdpp. $\boldsymbol{B}$, Decline of the mean cross-sectional area of rhabdomeres was compared with the intensity of Arr2-eGFP-labeled rhabdomeres from multiple ommatidia ("fluorescent dpp," see insets in $\boldsymbol{A}$ for depiction of $\mathrm{dpp})$. The age-dependent reduction of dpp and rhabdomere exhibit a similar time course. Data were normalized to 2-d-old norp $A^{P 24}$ and represent means \pm SEM $(n=4)$. C, The age-dependent decrease of the Rh1 level. Rh1, Arr2, and INAD were measured by Western blotting and expressed as a percentage of the 2-d-old flies of the same genotype. The Rh1 content was gradually diminished, while the Arr2 level remained constant in degenerating norpA ${ }^{P 24}$ flies. The level of INAD, a membrane-associated cytosolic protein, served as a positive control. INAD remains constant but starts to decline only at the later stages of degeneration in norpA flies. Values represent means $\pm \operatorname{SEM}(n=4),{ }^{*} p<0.05,{ }^{* *} p<0.01$.

complex results in degeneration of R1-6 photoreceptors (Alloway et al., 2000; Orem and Dolph, 2002; Chinchore et al., 2009). We introduced wt Arr2-eGFP transgene into the norpA $A^{P 24}$ genetic background to monitor the endocytosis of the Rh1/Arr2 complex in live male flies during the progression of degeneration.

First, we show that degeneration of norp $A^{P 24}$ photoreceptors is marked by the age-dependent progressive deterioration of rhabdomeres (Fig. 3A). The cross-sectional area of Arr2-eGFPlabeled rhabdomeres in R1-6 photoreceptors remained constant initially; however, as norp $A^{P 24}$ flies aged in a $12 \mathrm{~h}$ light/dark cycle, rhabdomeres became smaller and eventually disappeared at $\sim 9 \mathrm{~d}$ posteclosion (Fig. $3 A, B$ ). In contrast, the size of rhabdomeres in wt R1-6 cells remained constant for up to $28 \mathrm{~d}$ (data not shown). It is likely that deterioration of rhabdomeres in norp $A^{P 24}$ photoreceptors is caused by the enhanced turnover of Rh1, as Rh1 is the major membrane protein in rhabdomeres (Kumar and Ready, 1995).

We compared the progressive diminution of rhabdomeres with the decline of Rh1 level, which can be determined by West- 
ern blotting (Fig. 3C) and by detection of dpp. Dpp reflects the superposition of Rh1 autofluorescence in neighboring rhabdomeres of the compound eye (Franceschini, 1972), and can be easily observed when illuminated with blue light. Because Rh1 recruits Arr2 stoichiometrically following activation by light (Satoh et al., 2010), the level of Arr2-eGFP in the rhabdomere is likely proportional to that of $\mathrm{Rh} 1$. Therefore we measured the intensity of fluorescent (fdpp) from Arr2-eGFP signal in rhabdomeres as a way to estimate the Rh1 content (Fig. $3 A, B$ ). Indeed, the decline of fdpp in norp $A^{P 24}$ displayed a time course (Fig. $3 B$ ) similar to the reduction of Rh1 measured by Western blotting (Fig. 3C). Furthermore, the decrease of fdpp is parallel to the decline of crosssectional rhabdomere areas in degenerating norp $A^{P 24}$ retinas (Fig. $3 B$ ). Based on these findings, we conclude that deterioration of rhabdomeres in norp $A^{P 24}$ photoreceptors is accompanied by a progressive reduction of $\mathrm{Rh} 1$.

The decrease in Rh1 thus reflects its internalization and subsequently degradation. We investigated whether Arr2 is also degraded along with Rh1. If Arr2 is indeed degraded, its steady-state level may be reduced similar to that of Rh1. Surprisingly, we found that the levels of endogenous Arr2 actually increased by $14.4 \pm 4.1 \%(n=4, p<0.05)$ at day 6 posteclosion, while the Rh1 level (5.7 \pm $3.4 \%, p<0.01)$ almost completely disappeared at day 8 (Fig. 3C). Based on this finding, it appears that Arr2 assists the internalization of activated Rh1 without being degraded.

\section{Inhibition of CaMKII alone does not} lead to degeneration of photoreceptors Degeneration of norp $A^{P 24}$ photoreceptors is instigated by a lack of $\mathrm{Ca}^{2+}$-dependent feedback regulation, which fails to activate both RDGC (Steele et al., 1992) and CaMKII (Cho et al., 1991). To explore whether inability to activate CaMKII alone is responsible for initiating degeneration, we monitored dpp by bright-field microscopy and the Rh1 level by Western blotting in white-eyed ala; $c n$ bw in which CaMKII was inhibited by the overexpression of an inhibitory peptide (Griffith et al., 1993). We found that 10-d-old $a l a^{1} ; c n b w$ flies of either sex exhibited no signs of retinal degeneration as dpp was still visible while the Rh1 level was not significantly reduced compared with that of wt (data not shown). In contrast, norp $A^{P 24}$ displayed complete degeneration by $9 \mathrm{~d}$ posteclosion (Fig. 3).

We also examined the distribution of activated Rh1 monitored by wt Arr2-eGFP in live retinas of red-eyed wt (Fig. 4A)

D

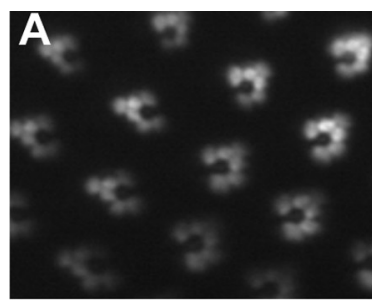

w+/y; Arr2-eGFP/+ 10-day

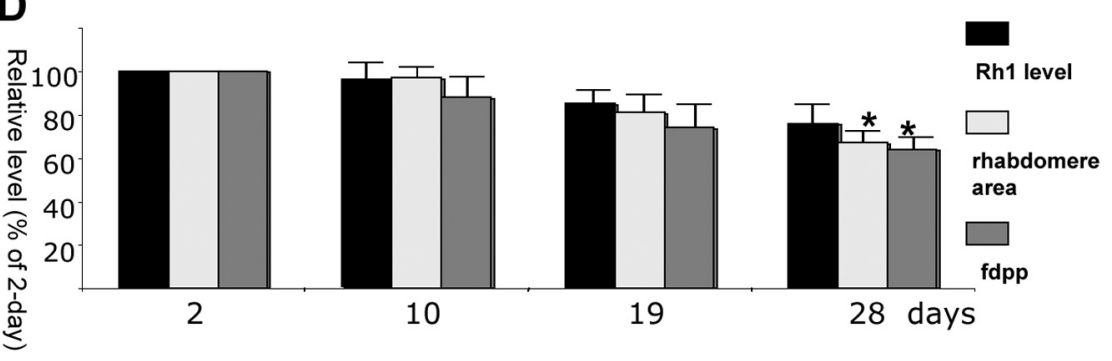

Figure 4. Suppression of CaMKII alone does not promote retinal degeneration. Morphology of rhabdomeres in ala ${ }^{1}$ flies overexpressing an inhibitory peptide for CaMKII was monitored by wt Arr2-eGFP for up to 4 weeks posteclosion. Ten-day-old wt control $(\boldsymbol{A}), 10$-d-old ala $^{1}(\boldsymbol{B})$, and 28-d-old ala $(\boldsymbol{C}) . \boldsymbol{D}$, Comparison of rhabdomere area, intensity of fdpp, and the Rh1 level in ala ${ }^{1}$ flies. Data were normalized as a percentage of the $2-d$-old flies of the same genotype and represent as mean $\pm \operatorname{SEM}(n=3) .{ }^{*} p<0.05$. At 4 weeks posteclosion, ala flies displayed a significant reduction in both rhabdomere area and intensity of fluorescent dpp but not in the Rh1 level, when compared with 2-d-old ala ${ }^{1}$ flies. All flies tested were red eyed. In red-eyed background, the shape of rhabdomeres usually appears more elongated, when compared with that of white-eyed flies (see Fig. 2).
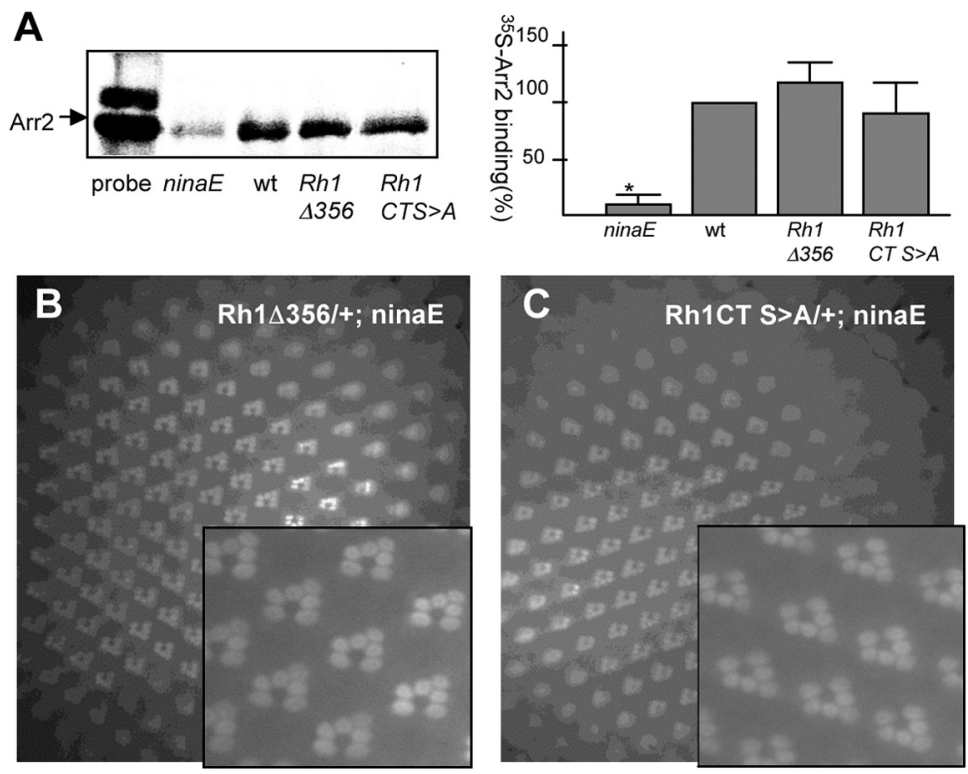

Figure 5. Arr2 binds to phosphorylation-deficient Rh1 in vitro and in vivo. A, Interaction between Arr2 and phosphorylationdeficient Rh1 in vitro by membrane binding assays. ${ }^{35} \mathrm{~S}$-Arr2 binding to either Rh1 $\Delta 356$ or Rh1CT S $>$ A was similar to ${ }^{35} \mathrm{~S}$-Arr2 binding to wt Rh1, whereas binding was greatly reduced when membranes prepared from ninaE ${ }^{117}$ were used. A representative autoradiograph depicting the bound ${ }^{35} \mathrm{~S}$-Arr2 is shown (top) and the results from three independent experiments are quantitated, normalized to binding to wt Rh1, and shown as means \pm SEM in the histogram. ${ }^{*} p<0.05$. $B$, The in vivo interaction between wt Arr2-eGFP and modified Rh1 lacking the $($ terminus, Rh1 $\Delta 356$, or the putative phosphorylation sites, Rh1CT S>A (C), was examined by fluorescence microscopy in 5-d-old live flies. Shown is a representative image from each interaction. and ala $^{1}$ flies (Fig. $4 B, C$ ). The presence of red pigment in the retina, which reduces the intensity of light stimulation, slows down the progression of the light-dependent degeneration of norp $A^{P 24}$ by $\sim 1$ week (our unpublished observations). We show that the cross-sectional areas of rhabdomere and the intensities of fdpp were not significantly affected in 10-d-old (97.3 $\pm 4.2 \%$; $88.5 \pm 10.6 \%, n=3)$ and 19 -d-old $(85.1 \pm 9.4 \% ; 78.8 \pm 15.2 \%$, 
$n=3$ ) ala ${ }^{1}$ flies (Fig. 4D); however, in 28-d-old ala ${ }^{1}$ flies both areas and fdpp intensity were decreased to $67.1 \pm 7.4 \%(n=3)$ and $64.4 \pm 8.3 \%(n=3)$, respectively, when compared with those in 2-d-old flies (Fig. 4D). Importantly, this reduction in rhabdomere area and fdpp intensity was not accompanied by a significant decrease of the Rh1 level $(80.4 \pm 13.5 \%, n=3)$ (Fig. $4 D$ ), and may be caused by a loss of phosphorylation in other CaMKII substrates that directly or indirectly impact the structure of rhabdomeres. Based on these results, we conclude that a loss of Arr2 phosphorylation by CaMKII appears not critical for initiating degeneration of norp $A^{P 24}$ photoreceptors as inhibition of CaMKII alone does not promote the internalization of Rh1.

Arr2 is able to interact with Rh1 independent of the C terminus or phosphorylation

It is very likely that a loss of $\mathrm{RDGC}$, the $\mathrm{Ca}^{2+}$-dependent protein phosphatase that controls dephosphorylation of Rh1 (Vinós et al., 1997), is responsible for orchestrating retinal degeneration in norp $A^{P 24}$. Because a loss of RDGC leads to an increased level of hyperphosphorylated Rh1 (Byk et al., 1993; Vinós et al., 1997; Kiselev et al., 2000), we hypothesized that phosphorylation of $\mathrm{Rh} 1$ is indispensable for photoreceptor degeneration by promoting the formation of a stable complex with Arr2.

We first examined whether phosphorylation is required for the Arr2 interaction. Previous studies have shown that the interaction between vertebrate visual arrestin and rhodopsin is regulated by phosphorylation of rhodopsin at its C terminus (Gurevich and Gurevich, 2004; Vishnivetskiy et al., 2007). Like vertebrate rhodopsin, light-dependent phosphorylation of Drosophila Rh1 also occurs at the $\mathrm{C}$ terminus, as a removal of the last 18 residues completely eliminates phosphorylation (Vinós et al., 1997). We used two transgenic lines expressing modified Rh1 lacking either the last 18 aa (Rh1 $\Delta 356)$ (Vinós et al., 1997) or the putative phosphorylation sites (Rh1CT S $>$ A) (Satoh and Ready, 2005). We performed metarhodopsin binding assays, and show that ${ }^{35} \mathrm{~S}$-Arr2 was able to bind to activated forms of these two phosphorylation-deficient Rh1s (112.2 $\pm 23.4 \%$; $81.3 \pm 34.3 \%$, $n=3$ ) similar to those of wt Rh1 (Fig. 5A). Moreover, we show that in live flies wt Arr2-eGFP is recruited to rhabdomeres containing Rh1 $\Delta 356$ (Fig. 5B) or Rh1CT S $>$ A (Fig. 5C).Together, these results support the notion that phosphorylation of Rh1 at its $\mathrm{C}$ terminus is not critical for the Arr2 interaction in vitro and in vivo.

Expression of phosphorylation-deficient Rh1 prevents, while co-expression greatly delays degeneration of $\operatorname{norp} A^{P 24}$ photoreceptors

To investigate whether interaction between phosphorylationdeficient Rh1 and Arr2 triggered light-dependent degeneration of norp $A^{P 24}$ photoreceptors, first we performed crosses by bringing two transgenes expressing modified Rh1s and wt Arr2-eGFP into the norp $A^{P 24}$ and ninaE $E^{I 7}$ double mutant background. Flies of desired genotypes were collected at eclosion to monitor the age-dependent degeneration. Significantly, we show that 20 -dold norp $A^{P 24}$ flies expressing Rh1 $\Delta 356$ (Fig. $6 A$ ) or Rh1CT S $>$ A (Fig. $6 B$ ) still contained the full complement of R1-6 rhabdomeres as visualized by wt Arr2-eGFP, and displayed no sign of retinal degeneration. These results strongly indicate that interaction between Arr2 and phosphorylation-deficient Rh1 does not lead to internalization of phosphorylation-deficient Rh1 and, consequently, retinal degeneration.

The interaction between phosphorylation-deficient Rhl and Arr2 generates Rh1/Arr2 complexes that are defective of internal-

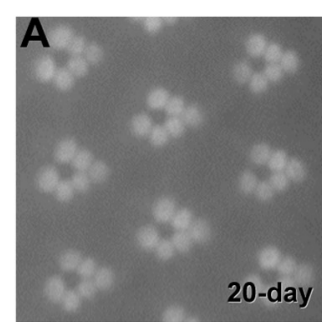

norpA; Rh1 $\triangle 356 ;$ ninaE

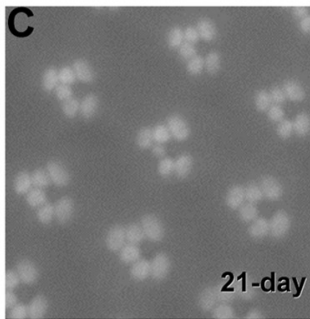

norpA;+/Rh1CT S>A; +/ninaE

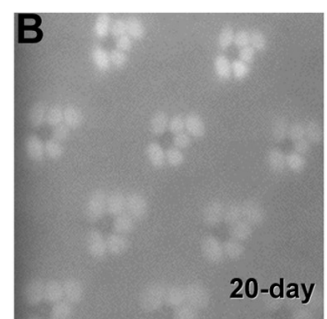

norpA;Rh1CT S>A; ninaE

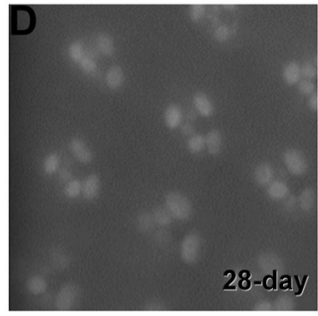

norpA;+/Rh1CT S>A; +/ninaE
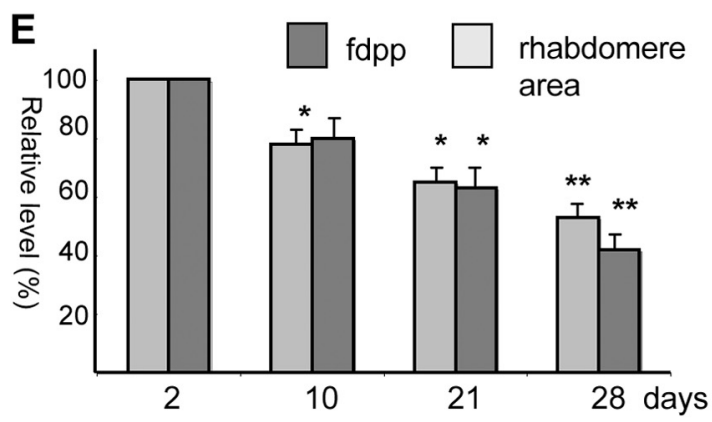

$\mathbf{F}$

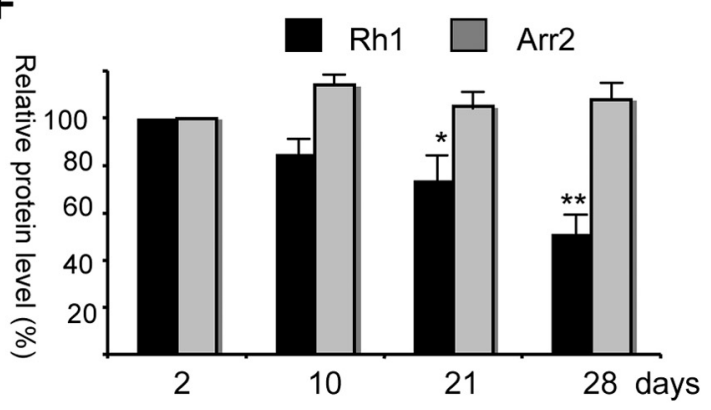

Figure 6. Expression of phosphorylation-deficient Rh1 prevents degeneration of norpA ${ }^{P 24}$ photoreceptors while co-expression greatly delays degeneration. Shown are retinal morphology monitored by wt Arr2-eGFP in norpA $A^{P 24}$ flies expressing either Rh1 $\Delta 356(\boldsymbol{A})$ or Rh1CT S $>$ A $(\boldsymbol{B})$ at $20 \mathrm{~d}$ posteclosion. Co-expression of Rh1CTS $>$ A greatly delayed degeneration $(\boldsymbol{C}$, at $21 \mathrm{~d}$; $\boldsymbol{D}$, at $28 \mathrm{~d}$ ). Comparison of rhabdomere areas and intensities of $\mathrm{fdpp}(\boldsymbol{E})$ and Rh1 levels $(\boldsymbol{F})$ of norpA $A^{P 24}$ flies co-expressing wt and Rh1CT S $>$ A. Data were normalized as a percentage of the 2 -d-old flies of the same genotype and represent as mean \pm SEM $(n=4) .{ }^{*} p<0.05$, ${ }^{* *} p<0.01$.

ization. It is possible that the formation of these internalizationdefective complexes may reduce the level of Arr2 in norp $A^{P 24}$ flies expressing both wt and phosphorylation-deficient Rh1. We monitored the time course of norp $A^{P 24}$ degeneration in male flies expressing one copy each of wt and Rh1CT S $>$ A. Indeed, we show that degeneration was delayed, as rhabdomeres retained wt-like morphology for at least 3 weeks posteclosion (Fig. 6C) while norp $A^{P 24}$ flies expressing two copies of wt Rh1 displayed complete degeneration by 2 weeks (Fig. 3). At 4 weeks, some ommatidia exhibited signs of deterioration characterized by missing and/or reduced rhabdomeres in norp $A^{P 24}$ flies coexpressing Rh1CT S>A (Fig. 6D). Detailed analysis indicated 


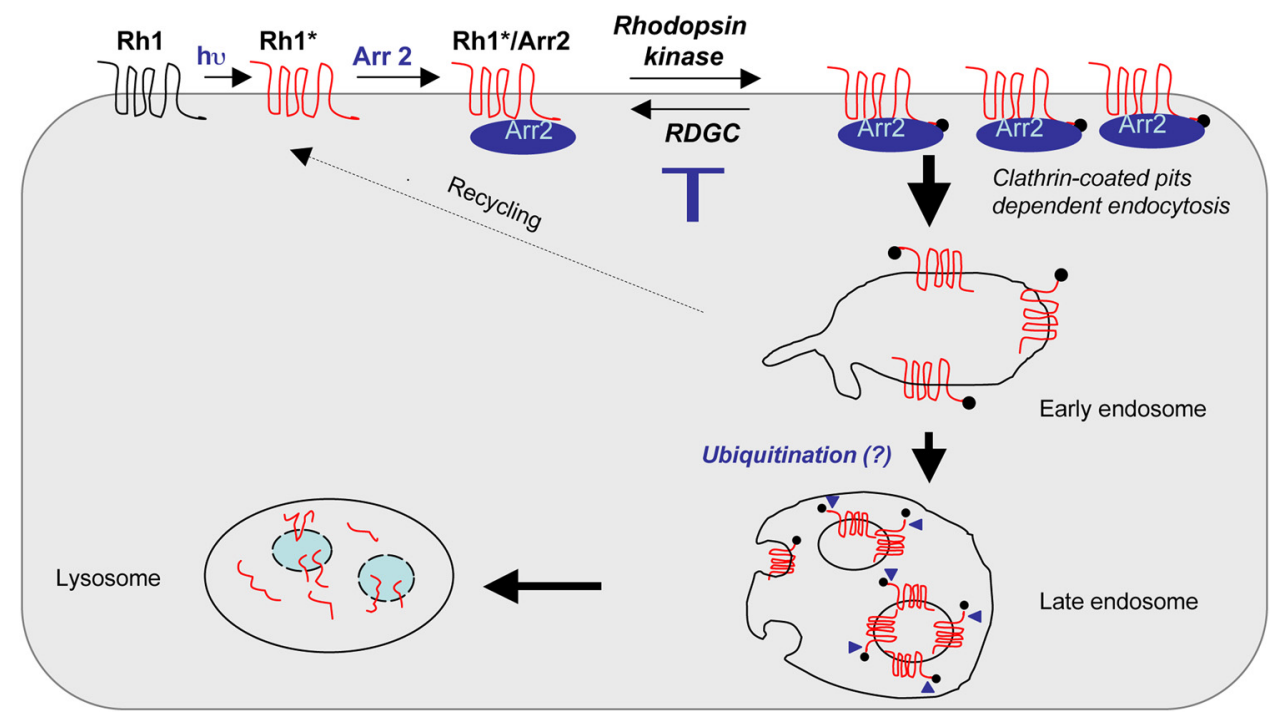

Figure 7. A hypothetical mechanism of enhanced internalization and degradation of phosphorylated Rh1 leading to retinal degeneration of norpA photoreceptors. In norpA photoreceptors, the lack of $\mathrm{Ca}^{2+}$ influx renders Rh1 phosphatase RDGC inactive resulting in an increased level of phosphorylated and activated Rh1 (Rh1*). Phosphorylated Rh1 forms stable complexes with Arr2 that promotes the endocytosis of Rh1* . Following internalization, Rh1* may be ubiquitinated and subsequently is trafficked to lysosomes for degradation. A reduction of Rh1 that is critical for the maintenance and function of photoreceptors results in loss of rhabdomeres and eventually, degeneration of photoreceptors. Filled circles denote phosphorylation at the C terminus of Rh1, and filled triangles denote ubiquitination of Rh1.

that the progression of degeneration as revealed in rhabdomere areas $(53.3 \pm 5.6 \%, n=4)$ and $\mathrm{fdpp}(42.5 \pm 6.1 \%, n=4)$ (Fig. $6 E)$ as well as the Rh1 level $(48.6 \pm 9.6 \%, n=4)$ is greatly delayed in 28-d-old flies (Fig. 6 F), when compared with norpA $A^{P 24}$ flies of the same age expressing two copies of wt Rh1 gene (Fig. 3).Together, we conclude that phosphorylation at the $\mathrm{C}$ terminus of $\mathrm{Rh} 1$ is required for triggering degeneration of norp $A^{P 24}$ photoreceptors, as modified Rh1s devoid of phosphorylation sites did not appear to be degraded and, consequently, degeneration was not observed.

\section{Discussion}

Reversible phosphorylation has commonly served to modulate diverse signaling processes. Dysfunction of these regulatory events often has detrimental consequences affecting cell survival and resulting in diseases. Here we provide evidence to firmly support the critical role of Rh1 phosphorylation, but not a lack of Arr2 phosphorylation, in retinal degeneration of norp $A^{P 24}$ flies. In Drosophila visual signaling cascade, Arr2 binding to activated Rh1 promotes its timely inactivation (Dolph et al., 1993; Ranganathan and Stevens, 1995). The interaction between Rh1 and Arr2 appears transient, and Arr2 is released when Rh1 becomes inactivated. This is partly achieved by $\mathrm{Ca}^{2+}$ dependent regulatory mechanisms, which include CaMKII (Matsumoto et al., 1994; Kahn and Matsumoto, 1997) and RDGC (Byk et al., 1993; Vinós et al., 1997; Kiselev et al., 2000). When the $\mathrm{Ca}^{2+}$ dependent regulation is rendered defective, the interaction between Arr2 and Rh1 may become more persistent (Alloway and Dolph, 1999; Alloway et al., 2000) leading to massive internalization of these complexes and ushering degeneration of photoreceptors.

It has been shown that massive endocytosis of the Rh1 complex in norp $A^{P 24}$ is controlled by Arr2 via its direct interaction with AP-2 (Orem et al., 2006) and/or clathrin (Kiselev et al., 2000), both of which are involved in the clathrin-dependent endocytic pathway (Robinson, 1994). The Arr2-clathrin interaction may be negatively regulated by Arr2 phosphorylation because phosphorylated Arr2 fails to associate with clathrin (Kiselev et al.,
2000). In other words, unphosphorylated Arr2 binds to clathrin, which may lead to degeneration. Phosphorylation of Arr2 at $\mathrm{Ser}^{366}$ is catalyzed by CaMKII (Matsumoto et al., 1994). However, we show that inhibition of CaMKII did not greatly perturb retinal morphology of ala $^{1}$ flies. This observation led to our conclusion that a loss of Arr2 phosphorylation does not provoke degeneration of norp $A^{P 24}$ photoreceptors.

In vertebrates, it has been well established that visual arrestins interact with phosphorylated and photoactivated rhodopsin. In Drosophila, however, we found that phosphorylation of Rh1 is not required for its interaction with Arr2, as Arr2 can associate with rhabdomeric membranes containing phosphorylationdeficient Rh1 that either lacks the $\mathrm{C}$ terminus or the putative phosphorylation sites. Our findings are consistent with previous reports (Kiselev et al., 2000; Satoh and Ready, 2005) and strongly suggest that reversible phosphorylation of Rh1 plays a novel role in controlling its activity and deposition from the membrane. Indeed, Vinós et al. (1997) reported that phosphorylation of Rh1 is required for fast inactivation of Rh1. Here, we demonstrate that phosphorylation of Rh1 is also involved in the Arr2-mediated degeneration, as expression of phosphorylation-deficient Rh1 completely blocked degeneration of norp $A^{P 24}$ photoreceptors. Our findings support the notion that phosphorylation of Rh1 is essential for the formation of the stable complex with Arr2, which, when occurring extensively, interferes with the maintenance and affects the survival of photoreceptors.

We monitored photoreceptor degeneration in live retinas, and showed that the gradual decrease of rhabdomere size is parallel to the reduction of Rh1. Our findings support the notion that a reduction of Rh1 due to enhanced turnover causes deterioration of rhabdomeres. Rh1 is known as the critical protein for orchestrating the morphogenesis of rhabdomeres during development. A decrease of Rh1 biosynthesis due to defects in translation and post-translational processing leads to reduced rhabdomeres and retinal degeneration (Leonard et al., 1992; Colley et al., 1995; Kurada and O’Tousa, 1995). Our results strongly suggest that a reduction of Rh1 
post development also negatively impacts the maintenance of rhabdomeres and photoreceptors.

Our findings are consistent with the current knowledge concerning the role of G-protein-coupled receptor (GPCR) phosphorylation in arrestin-dependent endocytosis in vertebrates (for review, see Moore et al., 2007). It has been well established that phosphorylation of GPCR and subsequent $\beta$-arrestin binding targets receptors to clathrin-coated pits for internalization (for review, see Ferguson et al., 1996). Internalized receptors are sorted in the early endosome, which are either trafficked to recycling endosomes for reinsertion into the plasma membrane or to multivesicular late endosomes for subsequent lysosomal degradation. Our current hypothesis regarding the fate of endocytosed and phosphorylated Rh1 is summarized in Figure 7. It is likely that phosphorylated Rh1 is ubiquitinated, which appears critical for trafficking of activated GPCRs to lysosomes (Shenoy et al., 2001).

$\beta$-arrestins are critical in modulating these endocytic events of GPCRs, as the affinity of the interaction dictates the endocytic processes of GPCRs for either recycling or degradation (for review, see Moore et al., 2007). Based on the affinity with $\beta$-arrestins, GPCRs can be subdivided into two classes, Class A and Class B (Oakley et al., 1999). Class A receptors show a transient $\beta$-arrestin interaction and rapidly dissociate from $\beta$-arrestin near or at the plasma membrane once internalized, while Class B receptors display a more stable $\beta$-arrestin interaction and are colocalized with $\beta$-arrestin in endosomes. A stable $\beta$-arrestin interaction requires a conserved cluster of Ser/Thr at the C terminus of the receptor, which is subject to phosphorylation by GPCR kinases (GRKs). Interestingly, different GRKs phosphorylate GPCRs differentially to modulate distinct endocytic trafficking events (Oakley et al., 2001). In Drosophila, the Rh1 interaction with Arr2 is reminiscent of the more transient interaction of the Class A receptor, as Arr2 likely dissociates from Rh1 near the membrane. Different from the Class A receptor, the endocytosed $\mathrm{Rh} 1$ is not recycled but is most likely degraded.

Select forms of retinal degeneration in humans may be contributed to by the formation of a stable complex between visual arrestin and photoactivated rhodopsin leading to the persistent endocytosis of photoactivated rhodopsin (Chen et al., 2006). In Drosophila, we show that phosphorylation of Rh1 is essential for Arr2-dependent retinal degeneration observed in norp $A^{P 24}$ photoreceptors. We show that expression of phosphorylation-deficient Rh1 completely blocks the degeneration, while co-expression greatly delays it. This partial rescue of degeneration provides a glimpse into mechanistic insights by which Arr2 promotes the endocytosis of Rh1. It appears that interaction between hypophosphorylated Rh1 and Arr2 results in the formation of endocytosis-incompetent complexes, which may decrease the level of functional Arr2 delaying the onset of degeneration. Thus, we propose that a potential therapeutic intervention for treating visual arrestin-dependent retinal degeneration is to partially reduce phosphorylation of rhodopsin by using selective rhodopsin kinase inhibitors.

In summary, we explored the contribution of reversible phosphorylation of Rh1 and Arr2 in mediating retinal degeneration of norp $A^{P 24}$ photoreceptors. We show that a reduced phosphorylation of Arr2 appears to have no overt consequence on retinal morphology, whereas hyperphosphorylation of Rh1 plays an integral role for initiating degeneration of R1-6 photoreceptors. Importantly, co-expression of phosphorylation-deficient Rh1 slows down degeneration, which may be applicable for treating human retinal dystrophy caused by persistent internalization of rhodopsin-arrestin complexes.

\section{References}

Alloway PG, Dolph PJ (1999) A role for the light-dependent phosphorylation of visual arrestin. Proc Natl Acad Sci U S A 96:6072-6077.

Alloway PG, Howard L, Dolph PJ (2000) The formation of stable rhodopsin-arrestin complexes induces apoptosis and photoreceptor cell degeneration. Neuron 28:129-138.

Byk T, Bar-Yaacov M, Doza YN, Minke B, Selinger Z (1993) Regulatory arrestin cycle secures the fidelity and maintenance of the fly photoreceptor cell. Proc Natl Acad Sci U S A 90:1907-1911.

Chen J, Shi G, Concepcion FA, Xie G, Oprian D, Chen J (2006) Stable rhodopsin/arrestin complex leads to retinal degeneration in a transgenic mouse model of autosomal dominant retinitis pigmentosa. J Neurosci 26:11929-11937.

Chinchore Y, Mitra A, Dolph PJ (2009) Accumulation of rhodopsin in late endosomes triggers photoreceptor cell degeneration. PLoS Genet 5:e1000377.

Cho KO, Wall JB, Pugh PC, Ito M, Mueller SA, Kennedy MB (1991) The alpha subunit of type II Ca2+/calmodulin-dependent protein kinase is highly conserved in Drosophila. Neuron 7:439-450.

Colley NJ, Cassill JA, Baker EK, Zuker CS (1995) Defective intracellular transport is the molecular basis of rhodopsin-dependent dominant retinal degeneration. Proc Natl Acad Sci U S A 92:3070-3074.

Dolph PJ, Ranganathan R, Colley NJ, Hardy RW, Socolich M, Zuker CS (1993) Arrestin function in inactivation of G protein-coupled receptor rhodopsin in vivo. Science 260:1910-1916.

Ferguson SS, Downey WE 3rd, Colapietro, AM Barak, LS Ménard L, Caron MG (1996) Role of beta-arrestin in mediating agonist-promoted G protein-coupled receptor internalization. Science 271:363-366.

Franceschini N (1972) Pupil and pseudopupil in the compound eye of Drosophila. In. Information processing in the visual system of Drosophila (Wehner R, ed.). Berlin: Springer.

Griffith LC, Verselis LM, Aitken KM, Kyriacou CP, Danho W, Greenspan RJ (1993) Inhibition of calcium/calmodulin-dependent protein kinase in Drosophila disrupts behavioral plasticity. Neuron 10:501-509.

Gurevich VV, Gurevich EV (2004) The molecular acrobatics of arrestin activation. Trends Pharmacol Sci 25:105-111.

Hardie RC (2007) TRP channels and lipids: from Drosophila to mammalian physiology. J Physiol 578:9-24.

Kahn ES, Matsumoto H (1997) Calcium/calmodulin-dependent kinase II phosphorylates Drosophila visual arrestin. J Neurochem 68:169-175.

Kahn ES, Kinumi T, Tobin SL, Matsumoto H (1998) Phosrestide-1, a peptide derived from the Drosophila photoreceptor protein phosrestin I, is a potent substrate for $\mathrm{Ca} 2+/$ calmodulin-dependent protein kinase II from rat brain. Comp Biochem Physiol B Biochem Mol Biol 119:739-746.

Kiselev A, Socolich M, Vinós J, Hardy RW, Zuker CS, Ranganathan R (2000) A molecular pathway for light-dependent photoreceptor apoptosis in Drosophila. Neuron 28:139-152.

Kumar JP, Ready DF (1995) Rhodopsin plays an essential structural role in Drosophila photoreceptor development. Development 121:4359-4370.

Kurada P, O’Tousa JE (1995) Retinal degeneration caused by dominant rhodopsin mutations in Drosophila. Neuron 14:571-579.

Leonard DS, Bowman VD, Ready DF, Pak WL (1992) Degeneration of photoreceptors in rhodopsin mutants of Drosophila. J Neurobiol 23:605-626.

Leung HT, Tseng-Crank J, Kim E, Mahapatra C, Shino S, Zhou Y, An L, Doerge RW, Pak WL (2008) DAG lipase activity is necessary for TRP channel regulation in Drosophila photoreceptors. Neuron 58:884-896.

Lu H, Leung HT, Wang N, Pak WL, Shieh BH (2009) Role of Ca2+/ calmodulin-dependent protein kinase II in Drosophila photoreceptors. J Biol Chem 284:11100-11109.

Matsumoto H, Kurien BT, Takagi Y, Kahn ES, Kinumi T, Komori N, Yamada T, Hayashi F, Isono K, Pak WL (1994) Phosrestin I undergoes the earliest light-induced phosphorylation by a calcium/calmodulin-dependent protein kinase in Drosophila photoreceptors. Neuron 12:997-1010.

Mishra P, Socolich M, Wall MA, Graves J, Wang Z, Ranganathan R (2007) Dynamic scaffolding in a $G$ protein-coupled signaling system. Cell 131:80-92.

Moore CA, Milano SK, Benovic JL (2007) Regulation of receptor trafficking by GRKs and arrestins. Annu Rev Physiol 69:451-482.

Oakley RH, Laporte SA, Holt JA, Barak LS, Caron MG (1999) Association of beta-arrestin with $\mathrm{G}$ protein-coupled receptors during clathrin-mediated 
endocytosis dictates the profile of receptor resensitization. J Biol Chem 274:32248-32257.

Oakley RH, Laporte SA, Holt JA, Barak LS, Caron MG (2001) Molecular determinants underlying the formation of stable intracellular $\mathrm{G}$ proteincoupled receptor-beta-arrestin complexes after receptor endocytosis* J Biol Chem 276:19452-19460.

Orem NR, Dolph PJ (2002) Loss of the phospholipase C gene product induces massive endocytosis of rhodopsin and arrestin in Drosophila photoreceptors. Vision Res 42:497-505.

Orem NR, Xia L, Dolph PJ (2006) An essential role for endocytosis of rhodopsin through interaction of visual arrestin with the AP-2 adaptor. J Cell Sci 119:3141-3148.

O’Tousa JE, Baehr W, Martin RL, Hirsh J, Pak WL, Applebury ML (1985) The Drosophila ninaE gene encodes an opsin. Cell 40:839-850.

Pak WL, Leung HT (2003) Genetic approaches to visual transduction in Drosophila melanogaster. Receptors Channels 9:149-167.

Ranganathan R, Stevens CF (1995) Arrestin binding determines the rate of inactivation of the $G$ protein-coupled receptor rhodopsin in vivo. Cell 81:841-848

Robinson MS (1994) The role of clathrin, adaptors and dynamin in endocytosis. Curr Opin Cell Biol 6:538-544.

Satoh AK, Ready DF (2005) Arrestin1 mediates light-dependent rhodopsin endocytosis and cell survival. Curr Biol 15:1722-1733.

Satoh AK, Xia H, Yan L, Liu CH, Hardie RC, Ready DF (2010) Arrestin translocation is stoichiometric to rhodopsin isomerization and acceler- ated by phototransduction in Drosophila photoreceptors. Neuron 67:997-1008.

Shenoy SK, McDonald PH, Kohout TA, Lefkowitz RJ (2001) Regulation of receptor fate by ubiquitination of activated $\beta 2$-adrenergic receptor and $\beta$-arrestin. Science 294:1307-1313.

Shieh BH (2011) Molecular genetics of retinal degeneration: a Drosophila perspective. Fly (Austin) 5 .

Shieh BH, Niemeyer B (1995) A novel protein encoded by the InaD gene regulates recovery of visual transduction in Drosophila. Neuron 14:201-210.

Steele FR, Washburn T, Rieger R, O’Tousa JE (1992) Drosophila retinal degeneration $\mathrm{C}(\mathrm{rdgC})$ encodes a novel serine/threonine protein phosphatase. Cell 69:669-676.

Vinós J, Jalink K, Hardy RW, Britt SG, Zuker CS (1997) A G proteincoupled receptor phosphatase required for rhodopsin function. Science 277:687-690.

Vishnivetskiy SA, Raman D, Wei J, Kennedy MJ, Hurley JB, Gurevich VV (2007) Regulation of arrestin binding by rhodopsin phosphorylation level. J Biol Chem 282:32075-32083.

Wang T, Montell C (2007) Phototransduction and retinal degeneration in Drosophila. Pflugers Arch 454:821-847.

Yang TT, Cheng L, Kain SR (1996) Optimized codon usage and chromophore mutations provide enhanced sensitivity with the green fluorescent protein. Nucleic Acids Res 24:4592-4593.

Zuker CS, Cowman AF, Rubin GM (1985) Isolation and structure of a rhodopsin gene from D. melanogaster. Cell 40:851-858. 